\title{
Philosophiques
}

\section{Spencer, le concept de société : entre organicisme et individualisme}

\section{Claude Gauthier}

Volume 20, numéro 1, printemps 1993

URI : https://id.erudit.org/iderudit/027202ar

DOI : https://doi.org/10.7202/027202ar

Aller au sommaire du numéro

Éditeur(s)

Société de philosophie du Québec

ISSN

0316-2923 (imprimé)

1492-1391 (numérique)

Découvrir la revue

Citer cet article

Gauthier, C. (1993). Spencer, le concept de société : entre organicisme et individualisme. Philosophiques, 20(1), 3-24. https://doi.org/10.7202/027202ar
Résumé de l'article

RESUMÉ

Il s'agit, à partir d'une étude de la double typologie des sociétés chez Spencer, de montrer qu'il y a, dans sa pensée, pluralité de modèles théoriques mobilisés au service d'une conception sociologique qui passe, généralement, pour organiciste. On tente alors de faire apparaître l'existence d'un pôle individualiste et de réévaluer la portée de son organicisme. En même temps, l'on s'attache à souligner le caractère très relatif de son évolutionnisme. d'utilisation que vous pouvez consulter en ligne.

https://apropos.erudit.org/fr/usagers/politique-dutilisation/ 


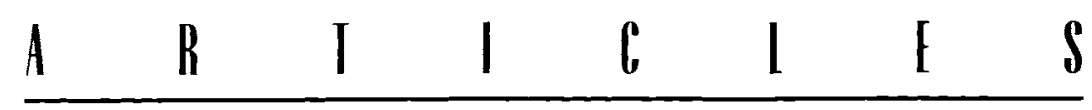

\title{
SPENCER, IE CONCEPT DE SOCIEIIE : ENTRE ORGANCLISME E E NDDHDUAALISME
}

\author{
par \\ Claude Gauthier
}

RÉSUMÉ : Il s'agit, à partir d'une étude de la double typologie des sociétés chez Spencer, de montrer qu'll y a, dans sa pensée, pluralité de modèles théoriques mobilisés au service d'une conception sociologique qui passe, généralement, pour organiciste. On tente alors de faire apparaitre l'existence d'un pôle individualiste et de réévaluer la portée de son organicisme. En même temps, l'on s'attache à souligner le caractère très relatif de son évolutionnisme.

ABSTRACT: This is a study of Spencer's double typology of societies. The goal is to show that Spencer put forward several theoretical models, within a sociological framework generally held to be organicist. The author brings out that one of the poles of this framework is individualistic. A reevaluation of Spencer's organicism follows, and his evolutionism is shown to be relative.

Les historiens de la pensée n'ont pas manqué de souligner l'influence souvent déterminante de l'évolutionnisme et de l'organicisme sur la conception spencérienne de la société. D’abord. une orientation évolutionniste qui est une donnée caractéristique des modèles sociologiques de cette «tradition » de la fin du 
$\mathrm{XIX}^{\mathrm{I}^{1}}$, ensuite une représentation organiciste dans la mesure où toute la sociologie de Spencer est traversee par ces analogies entre biologie et sociologie. Ne dira-t-il pas lui-mème que la société est un organisme ${ }^{2}$ ? Et pourtant, cette affirmation ne laisse pas d'être paradoxale. Une lecture attentive des Principes de sociologie ne permet pas de faire l'hypothèse d'une coherence globale de cette pensée : plusieurs modèles théoriques y sont mobilisés. Le problème semble résider dans la difficulté qu'il y a à affirmer que la société est un organisme et, en même temps, à reconnaître qu'« étant donné la nature des unités, celle des composés qu'ils forment est déterminée $»^{3}$.

Principalement, Spencer a recours à deux types de modéles : le modèle organiciste, le modèle individualiste. C'est là une première forme d'hétérogénéité que nous tâcherons de mettre en évidence; en elle-même, celle-ci ne nuit pas à la richesse de l'argumentation, au contraire. Nous montrerons qu'elle est un des moyens par lesquels Spencer restitue la complexité de son objet. La seconde forme d'hétérogénéité, moins évidente à déceler, tient dans l'usage qui est fait de ces deux modèles. En effet, si nous prenons le cas de l'organicisme, par exemple, nous en trouvons deux usages possibles : un usage proprement méthodologique par lequel l'analogie avec l'organisme signifie simplement que l'on considère la société comme une totalité organisée, ce point de vue permettant de rendre compte de phénomènes sociaux avec des méthodes conformes à l'idéal scientifique du savoir ; un usage ontologique par lequel la distance entre objet et modèle disparaît au profit d'une indistinction qui conduit à faire de l'objet la réalité du modèle. Les mêmes constats peuvent être faits à propos du modèle individualiste.

I. Nous nous référons à l'ouvrage classique de R.A. Nisbet, La Tradition sociologique, Paris, PUF, Ig84. L'auteur définit la « tradition » à partir de la réaction romantique aux excès de l'individualisme rationnel du Siècle des lumières. Cette caractérisation repose sur le principe des oppositions conceptuelles dont l'opposition communauté / socièté est un exemple.

2. Cest le titre du second chapitre de la seconde partie des Principes de sociologie. Nous renvoyons, pour la traduction française à l'édition suivante: Principes de sociologic, $3^{\prime \prime}$ èd., Paris, F. Alcan, r887, 4 t., trad. Cazelles et Gerschel.

3. Introductiona la science sociale; pour la traduction française, nous renvoyons à celle publièe à Paris, Librairie Germer Baillière, 1875 . 
Spencer aurait-il failli à cette exigence de méthode qui postule la nécessaire cohérence interne de la démarche? Nous ne prétendons pas résoudre définitivement ce problème; nous essaierons seulement de confirmer la légitimité des remarques précédentes à partir d'une analyse plus précise de la question du classement des sociétés.

Cette question, qui orienta en partie la célèbre polémique entre Spencer et Durkheim ${ }^{4}$, permet de dessiner très clairement les contours de ces incohérences dont nous faisons l'hypothèse. Cette réflexion sur la typologie des sociétés est récurrente dans toute la sociologie fondatrice et elle cristallise, à notre sens, de manière archétypique, les conflits de modèles théoriques. Spencer n'échappe pas, croyons-nous, à cette règle; mais la réponse qu'il donne est plus complexe, plus ambiguë que celle de Durkheim.

Spencer propose deux typologies : la première consiste à opposer « société simple » et « société composée », c'est-à-dire société homogène et instable, et société hétérogène et stable; la seconde, « société militaire » et « société industrielle». Elles sont données dans la seconde partie des Principes, intitulée «Inductions sociologiques ». Pourquoi deux classements? La question mérite d'autant plus d'être posée que ce qui frappe est la diversité des principes classificatoires retenus. Curieusement, seule la seconde typologie a fait l'objet d'études et de commentaires ${ }^{5}$. Il semble pourtant, nous essaierons de le montrer, qu'il n'est pas possible de comprendre les principes de construction liées à la seconde typologie sans avoir établi les liens qui unissent les deux classements.

Voici donc l'hypothèse qui guidera l'ensemble de cette réflexion : dans sa construction des classements, Spencer a

4. Nous renvoyons le lecteur à $D e$ la division du travail social, notamment au livre I, chapitre 4 : «Solidarité organique et solidarité contractuelle », p. I77 sq.

5. Même si Durkheim évoque implicitement l'opposition entre société simple et société composée pour caractériser les deux types de solidarité, c'est surtout la seconde typologie qui retiendra son attention. J. Caseneuve, dans son article de la R.F.S., 196I, II, 2, 48`53, fait une ètude assez dètaillée des sociétés industrielles et militaires chez Spencer sans évoquer une seule fois l'opposition entre société simple et sociète composee. Son article s'intitule d'ailleurs « Socièté industrielle et société militaire ». 
recours, de manière plus systematique, au modèle individualiste. La première typologie pourra être interprétée comme le résultat d'une transposition partielle de l'hypothèse jusnaturaliste des deux états : état de nature, état de société. Ce sera aussi pour Spencer le moyen de fonder sa vision individualiste de la société : le principe de différenciation permet de poser l'individu comme unité anthropologique première. La seconde typologie a pour objet de théoriser le passage d'une société où la totalité détermine les parties composantes vers une société oủ les parties déterminent la totalité qu'ils composent. Autrement dit, pour user de catégories plus courantes en sociologie, nous pouvons dire que la seconde typologie reconstruit le passage d'une société définie d'un point de vue holiste vers une société définie par le point de vue individualiste 6 .

\section{I. l'organicisme de Spencer est-il ontologique ou méthodologique?}

Pour W. Stark ${ }^{7}$, Spencer est le grand représentant de ce qu'il appelle la « forme positive » de l'organicisme ${ }^{8}$. Il serait possible de décrire la pensée sociologique en partant de trois modèlestypes : l'organicisme, le mécanicisme ${ }^{9}$ et l'historicisme. Il existerait une différence principale entre l'historicisme et les deux premiers modèles : « La physiologie et la physique sont des modèles inadéquats dans leur utilisation en science sociale parce que ces sciences ont pour objet une réalité que l'homme découvre, et non

6. Nous renvoyons à la distinction dumontienne holisme / individualisme, celle qu'il définit dans Homo Aqualis et qu'il reprend dans ses Essais surl'individualisme.

7. W. Stark, The Fundamental Fonms of Social Thought, New York, Fordham University Press, Ig63.

8. W. Stark distingue notamment l'organicisme normatif qui n'a pas pour prétention de décrire le réel, mais qui, par élaboration d'un modèle « idéal », permet de définir un terme par rapport auquel il devient possible de comparer ce réel, et d'indiquer une orientation « normative » à l'évolution de celui-ci. L'organicisme positif se caractériserait, lui, par une prétention : celle de décrire objectivement la réalité grâce à l'analogie avec l'organisme.

9. W. Stark oppose organicisme et mécanicisme en ce que la référence principale du second est constituée par la science physique. Plus exactement, son support naturel est la mécanique rationnelle. Dans ce cas, pour la sociologie de type " mécaniste », si l'individu est posée comme réalité première, alors la cohérence interindividuelle à l'échelle de la société sera pensée comme résultante de forces individuelles. 
une réalité qu'il construit - une réalité qu'il ne peut pas comprendre dans ses origines et dans sa formation ${ }^{\text {IO }}{ }^{\text {. Le modele }}$ historique présenterait donc l'avantage de poser d'emblée la question de la production de la sociéte par les hommes.

Stark, à propos de Spencer, souligne à juste titre une première difficulté : dans l'affirmation « la société est un organisme », y a$t-i l$ identification entre les deux substantifs ou simplement analogie? En d'autres termes, Spencer fait-il un usage méthodologique de l'analogie, se contentant de trouver dans la société des analogues, lesquels permettent d'élaborer un point de vue scientifique sur la société ? Ou bien est-ce la confusion / identification entre les deux - la société est un organisme -, qui est envisagée, ce qui reviendrait à « réifier » ce qui découle d'une hypothèse de méthode, à savoir l'analogie. Stark cherchera à montrer que Spencer, tout en évoquant des différences notables entre organismes biologique et social, minimise leurs significations pour tendre vers l'affirmation d'une identité. Nous ne partageons pas ce point de vue. Si Spencer ne fait pas consciemment un usage méthodologique de l'analogie lorsqu'il présente sa double typologie - ce qui donnerait raison à $W$. Stark -, il n'en reste pas moins vrai que les conclusions auxquelles il parviendra (infra) ne peuvent être justement évaluées si nous ne faisons pas une telle hypothèse.

C'est donc par analogie avec les organismes vivants que Spencer définit, dans la seconde partie des Principes de sociologie, les caractères de la société. L'ensemble du texte est organisé autour d'un principe comparatif : l'étude détaillée des organismes biologiques permet de faire ressortir des singularités susceptibles de transposition; on en peut trouver les analogues dans l'organisme social. Mais cette première étape de la démarche est complétée par une étude des dissemblances ${ }^{\mathrm{II}}$.

Io. W. Stark, op. cit., p. 12 : «Physiology and physics are spurious models in the social sciences because they are concened with a reality which man finds, not with one which he makes $-a$ reality which he cannot understand in its inner origination and working ".

II. Si J.E. Schlanger rejoint Stark sur la qualification de l'usage spencérien de l'analogie, nous serons amené à penser, toutefois, que la nature des dissemblances envisagées, l'usage qui en sera fait dans la cinquième partie des Principes consacrée à l'étude des institutions politiques, doivent permettre de relativiser cette affirmation. J.E. Schlanger, Ies métaphores de lorganisme, Paris, Vrin, 1971. 


\section{Les analogies}

Celles-ci peuvent être résumées dans la proposition suivante: la société, comme l'organisme, vit en se divisant et en intégrant ses parties ainsi différenciées. Ce qui revient à dire que le principe de la division du travail, transposé de la division du travail biologique, est la marque de toute société, sans distinction de niveau de complexité. Cette division est alors coextensive à toute forme d'association, « la division du travail l...l est le fait qui constitue la société comme l'animal, à l'état de corps vivant $\gg[\S 217]$. Alors que pour Durkheim la division du travail est posée comme un facteur d'évolution des sociétés, pour Spencer, celle-ci permet de démarquer la société en général de ce qui n'est pas vivant. Autrement dit, même les sociétés «primitives », « peu évoluées » sont caractérisées par une division du travail. Nous retrouvons ici un des résultats de l'application de la loi générale d'évolution.

\section{Les dissemblances}

Elles sont de deux ordres. Tout d'abord, la société, à la différence du pur vivant, est un tout discret. Les points de contact entre les parties composantes ne sont donc pas de nature organique, mais bien plutôt de nature symbolique et culturelle:

l...l et quoique les membres d'un organisme social, qui ne forment pas un tout concret, ne puissent entretenir la coopération par des moyens matériels dont l'action se transmette d'une partie sur l'autre, ils ne laissent pas d'avoir le pouvoir de l'entretenir par une autre cause et de réaliser cet effet. Ils ne sont point en contact et pourtant ils agissent l'un sur l'autre à travers l'espace qui les sépare, par le langage de l'émotion et par le langage, oral et écrit, de l'intelligence » [\$ 22I].

Les parties entrent donc en contact à l'aide d'une médiation qui distingue irréversiblement la société de tout ensemble vivant, la culture comme artefact. Ainsi, Spencer, tout en voulant restreindre la portée de cette première dissemblance, la renforce en exhibant une dimension proprement humaine de la société. Cette démarcation, même relativisée dans le propos immédiat, ne manquera pas de jouer un rôle majeur dans l'élaboration des classements de sociétés. La seconde dissemblance introduit explicitement un des axiomes de l'individualisme comme mode de représentation de la société et comme méthode.

Chez les uns lles vivantsl, la conscience se concentre dans une petite part de l'agrégat. Chez les autres [les sociétésl, elle se trouve 
répandue partout dans l'agrégat : toutes les unités possèdent l'aptitude au bonheur et au malheur, sinon au même degré, du moins à des degrés voisins. Puisque donc il n'y a pas de sensorimm social, il s'ensuit que le bien être de l'agrégat, considéré à part des unités, n’est pas une fin qu'il faille chercher » [§ 220l.

Cette citation appelle plusieurs commentaires.

Tout d'abord. Spencer oppose l'organisme vivant à l'organisme social : dans le premier se trouve une distinction marquée entre organes moteurs, localisés, et organes sensoriels, périphériques; dans le second, il n'y aurait pas de distinction à faire entre organes moteurs et sens périphériques. Cette opposition peut être interprétée de la façon suivante. Une première formulation pourrait opposer une conscience unique à des organes sensoriels multiples (Descartes, VI" Méditation Métaphysique). Une seconde formulation pourrait être trouvée dans la réponse leibnizienne : chaque partie du corps est un organe sensoriel et une conscience. Est établie entre les deux une véritable solidarité: point de dualité au sein d'un être donné, seulement une transition insensible qui y relie le corps à l'âme. Nous reconnaissons ici une définition possible de la monade ${ }^{\mathrm{I} 2}$. Labsence de sensorium social permet à Spencer de proposer une autre définition de la société comme ensemble de monades, ou encore, comme ensemble d'individus. Mais cette citation contient une autre affirmation d'importance pouvant conduire à une représentation individualiste de la société : puisque toutes les parties composantes sont identiques en ce qu'elles allient une conscience et un organe sensoriel, elles sont égales devant l'aptitude au bonheur ou au malheur.

Cette seconde dissemblance conduit Spencer à admettre la possibilité d'une définition de la société sur la base du modèle individualiste : au départ, il y a des unités composantes égales, ou individus, et la société n'a pas à être distinguée de ses parties. Ou encore, faisant reposer ce nouveau concept de société sur l'axiome principal d'égalité, il ne pouvait que renverser le

12. "Si nous voulons appeler Âme tout ce qui a perceptions et appétits dans le sens général. que je viens dexpliquer, toutcs les substances smuples ou Monades créces pourraient cotre appelées Ämes (...) », § Ig, Leibniz, La Monadologit, Ed. Delagrave, présentation et notes par E. Boutroux, se reporter, notamment aux pages $60 \mathrm{sq}$. : « B. L'âme et le corps 》. 
corollaire lié au point de vue organiciste : la détermination des parties par le tout. Nous retrouvons ici une idée qu'il développera dans son Introduction à la science sociale et qui fondait les principes d'un individualisme de méthode: « étant donné la nature des unités, celle des composés qu'ils forment est déterminée », ou «qu'en d'autres termes l'agrégat présente une série de propriétés déterminées par la série des propriétés de ses parties et que la relation entre les deux séries constitue la matière d'une science $»^{13}$. Mais Spencer va beaucoup plus loin lorsqu'il envisage la possibilité d'une contradiction entre les données de l'action individuelle et les conséquences qui peuvent en résulter sur le plan de la totalité : l'histoire prouve « que les moyens mis en œuvre produisent presque toujours des résultats absolument imprévus $\gg^{14}$. Comment ne pas faire ici le rapprochement avec ce que Hayeck entend par spécificité des sciences humaines : « Les problèmes qu'elles lles sciences socialesl essaient de résoudre se présentent seulement dans la mesure où l'action consciente de nombreux individus produit des résultats inattendus, où des régularités sont observées qui ne sont pas le résultat d'un dessein personnel ${ }^{15}$.»

Ainsi, l'étude de la seconde dissemblance semble aller de pair avec un changement de modèle théorique : il n'est pas possible de pousser plus loin l'analogie entre organisme et société parce que si l'on veut en saisir véritablement la spécificité, il conviendrait de changer de modèle théorique. Lorsque J.E. Schlanger affirme que Spencer recherche avant tout des analogies réelles entre société et organisme, il n'en reste pas moins vrai que la définition de cette seconde dissemblance implique beaucoup plus que le simple constat d'une différence. Et si Spencer, dans le chapitre X de la seconde partie des Principes, n'y donne pas plus d'importance, il sera obligé d'en tenir compte lorsqu'il en viendra à définir ses typologies.

Nous pouvons conclure cette première partie en disant qu'il y a vraisemblablement un usage confus, pour le moins ambigu,

13. Introduction à la scicnce socialc, p. 53 et p. 55.

14. Op.cit.s P. 57.

15. Scientisme et sciences sociales, F. Hayeck, Paris, Plon, Col. Agorn, 1953, p. 53. 
du modèle organiciste chez Spencer. Mais, par cette nécessité implicite du changement de modele : de l'organicisme à l'individualisme, il semble mettre en évidence une des limites attachées à l'organicisme. Il convient, à un moment donné, pour penser les relations entre les parties, d'user d'un autre modèle théorique par lequel les interactions deviennent représentables comme système de forces. Tous ces premiers chapitres des «Inductions sociologiques » sont travaillés par cette double tension entre usages méthodologique et ontologique des modèles organiciste et individualiste.

\section{La première typologie ou l'opposition entre société simple et société complexe}

C'est dans les $\S \S 256$-257 que Spencer expose le contenu de cette première typologie. Cela le conduit à énoncer un principe nécessaire et universel à propos des phénomènes sociaux : « Les phases de composition et de recomposition sont des degrés où la société doit passer successivement » [§ 257]. Si le contenu évolutionniste de ce propos ne souffre aucune objection, ce résultat offre un autre intérêt, celui de comprendre les principes qui permettent à Spencer de poser une telle conclusion ${ }^{\mathrm{I}}$.

Il existe un point de vue à partir duquel on peut lire autrement cette première classification : sa finalité n'est pas de proposer une explication de la genèse historique des sociétés. La précision des exemples empruntés aux récits des voyageurs n'infirme pas cette hypothèse. Spencer lui-même en avait conscience qui tenait à préciser en fin d'analyse qu'« il ne faut pas prendre cette classification pour autre chose qu'une ébauche grossière par laquelle nous essayons d'approcher la vérité. Dans certains cas, les données fournies par les voyageurs et autres auteurs sont

I6. Par où il est démontré l'impossibilité d'une utilisation trop stricte des modèles de pensée en sociologie. Si W. Stark a raison de distinguer les formes organiciste, mécaniste et historique des sytèmes de pensée en sociologie, il est plus discutable dans son classement des auteurs de la tradition : Spencer, tout en étant organiciste, concilie la possibilité d'un raisonnement de type historique (il faudrait sur ce point étudier plus précisément le contenu de son évolutionnismel. Autrement dit, Stark aurait tort de vouloir assigner un auteur à un seul modèle. Nous retrouvons ici l'hypothèse selon laquelle il ne semble pas possible d'affirmer l'homogènéité des modèles théoriques sous-jacents à une pensée. 
insuffisantes, dans d'autres, leurs récits sont contradictoires » \§ 257]. Quelle est donc l'utilité méthodologique d'une typologie dont les fondements et les principes de classification reposent sur des données confuses, sujettes à caution? Plus, il semble que ce qui peut donner qualité à une typologie, sa valeur opératoire, n'est pas l'essentiel de ce qui retient l'attention de l'auteur.

L'hypothèse que nous défendrons est la suivante : cette typologie n'a pas pour objet de repérer empiriquement les sociétés et leurs transformations ; bien plutôt, elle cherche à définir les contours d'une « histoire idéal-typique » des sociétés ${ }^{17}$ et de leurs transformations; il s'agit d'un renouvellement de l'interrogation inaugurale $^{\mathrm{I} 8}$ sur la définition de la société. Les premières analogies sont retravaillées pour donner une réponse plus complexe. Celle-ci sera thématisée à travers l'opposition centrale entre société simple / société composée. Nous distinguerons l'analyse qui en est faite dans la seconde partie des Principes de sociologie, de celle donnée dans la cinquième partie.

\section{Société simple et société composée dans la seconde partie des Principes de sociologie}

Que faut-il entendre par société simple? Dès le départ, Spencer avoue le caractère idéal-typique de la démarche et de ses résultats : il n'est pas évident de repérer, dans la réalité, ce qu'il s'agit de définir, « en effet, comme dans tous les produits de l'évolution en général, les sociétés offrent des phases de transition qui se refusent à des séparations nettes » lop.cit.]. Ce qui ne l'empêche

17. Nous pouvons faire référence à Rousseau qui, dans son Second Discours, commence « par écarter tous les faits ». Remarquons que cette orientation proprement méthodologique de la démarche r'exclut pas, loin de là, des « incohérences », ou plutôt des confusions qui amènent Rousseau, comme Spencer que nous étudions ici. à prendre pour réel ce qui n'est que le résultat d'une démarche purement theorique.

I8. Spencer a été un lecteur assidu d'auteurs de la tradition moraliste anglaise du XVIII' siècle: A. Smith, A. Ferguson, etc. Il a eu l'occasion, en outre, de se familiariser avec les grands auteurs de l'école du droit naturel. L'interrogation inaugurale à laquelle nous renvoyons ici est bien celle qui, dans la tradition jusnaturaliste. consiste à poser la question de la légitimité de la société par la fameuse hypothèse des deux états : état de nature, état de société. Rappelons qu'il s'agit d'une hypothèse et non d'une généalogie de type historique. Elle permet seulement de fonder. sur le plan théorique, la nécessité de la socièté. 
pas de donner une définition : «comme société simple lelle est] celle qui forme un tout non assujetti à un autre et dont les parties coopèrent avec ou sans centre régulateur, en vue de certaines fins d'intérêt public » lop.cit. p. 136].

Deux idées forces sont à retenir : l'absence d'assujettissement. la question de la finalité de l'association. Ces deux idées ne conduisent-elles pas à définir un état présocial ? La société simple est d'abord une association contingente d'individus caractérisée par une extrême homogénéité. Les interactions individuelles y sont aléatoires et sporadiques. Ce qui contient les conflits tient dans des facteurs extérieurs : climat, localisation géographique, etc. Enfin, la société simple n'est pas encore une totalité organisée, elle n'est pas une complexité élaborée par laquelle le tout ne serait plus superposable aux parties. Il y manque l'hétérogène qui produit la différenciation. La société simple est ensuite une société où il n'y a pas grand sens à distinguer intérêts particuliers et intérêt public. Les deux finalités, individuelle et collective, sont indistinctes, non parce que la seconde contient la première et la comprime, mais parce qu'il n'y a pas de totalité sociale réellement constituée. Il est donc possible de définir la société simple comme association contingente d'individus qui, de façon aléatoire et instable, permet à chaque membre de pourvoir à ses besoins limités et peu élaborés. Nous pourrions parler plus justement d'état présocial.

La voie de passage de la société simple vers la société composée est suggérée par l'hypothèse de la formation et de la stabilisation de l'autorité politique : c'est par elle que débute le processus de la différenciation sociale ${ }^{19}$. Cette hypothèse sera reprise et développée dans la V" partie des Principes (infra). Ce passage du simple au composé permet donc à Spencer de démarquer état présocial et état social stable. Ceci appelle plusieurs remarques.

Il semble que cette première typologie, envisagée comme transposition de la loi d'évolution à l'étude des sociétés, permette

I9. Il est possible, ici, de remarquer le caractère tout à fait organiciste de la démarche : l'existence d'un centre nerveux décideur par quoi s'organise tout le processus de la différenciation sociale. 
de reformuler une hypothèse classique : dans un cadre théorique différent, il s'agit d'esquisser l'analogue des deux états : état de nature et état de société.

Le concept de société simple fonctionne comme hypothèse, comme abstraction théorique permettant, par opposition, de dégager les caractères de la société réelle ou « composée »: coopération, différenciation, organisation politique et stabilitée ${ }^{20}$.

Ce n'est donc pas comme essai d'étude anthropologique et historique que cette réflexion sur la société simple a une valeur, mais bien comme fiction logique et hypothétique par laquelle il devient possible d'envisager les formes réelles de toute association véritable.

L'étude de la société composée devrait faire accéder à l'analyse empirique. Ce n'est pas le cas. D'une part, Spencer pense que toute société réelle est composée, quoique la complexité de cette composition peut varier, ou, ce qui revient au même, qu'aucune société réelle ne peut être simple. Mais d'autre part, pour comprendre les sociétés réelles, il faut construire des modèles: le modèle logiquement élémentaire est celui de la société simple, auquel rien de réel ne correspond. Spencer devrait, à partir de ce modèle élémentaire, construire des modèles composés. Par là, il devrait enfin conclure sur la pertinence empirique des dits modèles. Or, la confusion conduit Spencer à présenter comme succession historique - évolutionnisme - ce qui n'est qu'engendrement logique : de l'élémentaire au composé ${ }^{2 \mathrm{I}}$.

Le jeu de la transposition de la loi d'évolution impose à Spencer une vision par étapes où la succession spatio-temporelle des états passe par des degrès de complexité croissants. Le souci excessif de classification le contraint, artificiellement, à envisager tous les cas possibles. Nous retrouvons ici l'indistinction classique (typique du dix-huitième siècle) qui présente comme une priorité historique ce qui n'est que la priorité épistémologique du modèle sur le modélisé.

20. Nous simplifions volontairement le problème, car nous pourrions montrer que Spencer n'échappe pas à l'ambiguité que nous trouvons aussi chez Rousseau : ou bien tout est idéal-typique, ou bien tout parle des sociétés réelles.

21. Par où l'évolutionnisme de Spencer serait rhétorique s'il était clair qu'il ne s'agissait que d'un mode d'exposition, ce qui n'est pas toujours le cas. 
Cette réutilisation du modèle hypothétique présente un autre intérêt : elle va permettre de placer l'origine des causes du changement à l'intérieur même de la société composée. Les principes de différenciation liés à la formation du politique inscrivent la société dans un mouvement, une histoire (?) qui est celle de sa complexification. Elle est nourrie par la loi d'hétérogénéité croissante qui conduit au principe d'intégration et donc à la transformation.

\section{Société simple et société composée dans la cinquième partie des Principes de sociologie}

La cinquième partie des Principes, consacrée à l'analyse de la genèse et du développement des institutions politiques, est l'occasion pour Spencer de revenir à la première typologie. Dans le quatrième chapitre، « Des forces et des formes politiques », Spencer pose à nouveau la question de savoir comment se réalise le passage de la société simple à la société composée, soit de la socièté indifférenciée à la société où se forment les premiers éléments de l'organisation politique.

Si les sociétés se sont développées, et si la dépendance mutuelle qui relie les parties, dépendance que suppose la coopération, s'est effectuee graduellement, il faut admettre que, en dépit des dissemblances qui finissent par sépirer les structures développées, il y a toujours une structure rudimentaire d'où toutes sortent. Enfin, si nous pouvons reconnaître cette unité primitive, la constatation de ce fait nous aidera à interpréter la diversité finale ltome III, p. 4zol.

Ce que Spencer appelait société simple, dans la seconde partie des Principes, est ici défini sous une forme différente. Il s'agirait de cette unité sociale primitive, universelle parce que repérable en toute forme sociale complexe. Unité primitive, car le complexe ne serait qu'une intégration - au sens mathématique-de la diversité des relations et combinaisons possibles de ces mêmes unités. Dans cette partie de l'ouvrage, la société simple est donc entendue comme l'élément indivisible, la monade, à partir de quoi, par effet de « composition », s'élabore la société réelle ${ }^{22}$. Par analogie, bien sûr, cette unité se rapproche de la cellule.

22. Nous retrouvons ici la même ambiguïté ou la même indistinction entre société composée comme modélisition du réel et société reelle. 
La transposition de l'hypothèse classique des deux états implique donc un second déplacement : l'état présocial indifférencie est envisagé comme composante de toutes les sociétés réelles. Mais s'il s'agit d'une fiction théorique permettant de construire la première typologie, dans la cinquième partie, celleci n'en désigne pas moins une réalité anthropologique simple qui peut être indifféremment l'individu ou la horde - la monade.

Comment s'explique alors le passage de cette entité abstraite et universelle au jeu concret de la différenciation par le biais de laquelle s'établit la société composée? Si nous retenons l'hypothèse de la seconde partie des Principes, il suffit d'appliquer la loi générale de l'évolution ou principe de la division du travail.

L'étape suivante de l'argumentation expose l'ensemble des causes qui provoquent la différenciation par quoi en vient à se former la première organisation politique. Spencer part de l'unité primitive originelle où, naturellement, vont s'exprimer les différences liées aux complexions psychologiques et morales des individus. Il y a des tempéraments qui se démarquent, et ces démarcations conduisent, à l'occasion d'événements contingents, aux premières scissions; puis ces scissions se stabilisent et engendrent des formes. Tel est le processus de composition de la société. Autrement dit, la société simple, comme état abstrait indifférencié est une société sans mouvement. Il est donc nécessaire d'admettre que, dans la réalité, chacun se valorise par des qualités et des aptitudes différentes, inégales. Ces inégalités engendrent le mouvement par lequel se développe la société composée. L'usage du principe de différenciation, qui n'est que la loi d'évolution sous une autre forme, traduit logiquement et rationnellement le mouvement par où la société existe comme ensemble évolutif.

La conséquence est donc la suivante: « D'une part, nulle force gouvernementale n'existe d'abord, sauf celle de la volonté commune exprimée par la horde assemblée. D'autre part, le rôle principal dans la détermination de cette volonté commune sera inévitablement joué par les hommes en petit nombre dont la supériorité est reconnue ${ }^{23}$.» Tel est le sens de la dernière 
détermination abstraite de la société simple : elle est volonté passive, c'est-à-dire volonté non développée qui n'a pas encore le mouvement en elle. Elle est contingentement manifestée par le fait d'une association informe, la horde. Le mouvement ou la mobilisation active des volontés est coextensif à la différenciation des individus. Plutôt, les différences naturelles vont d'abord se manifester par le jeu de volontés distinctes et l'expression de ces volontés sera d'abord le fait d'une minorité.

La société simple peut être définitivement conçue comme unité primitive indifférenciée, où les relations interindividuelles sont de caractère instable et non nécessaire, non agies par une volonté active. La mise en mouvement qui, à l'origine, fait correspondre volonté d'une minorité et volonté de tous permet à Spencer d'affirmer que ce n'est pas d'abord le modèle de la société individualiste de type démocratique qui vient à l'existence. Cette question des liens entre les volontés ne relève plus de la première typologie, mais bien de la seconde. Par où s'exprime la relation nécessaire entre les deux classements.

Finalement, cette première typologie énoncée conformément aux présupposés de la conception évolutionniste de Spencer, qui renvoie à une définition organiciste de la société, ne peut être convenablement étudiée sans accorder une place importante aux aspects du raisonnement de type individualiste qui en expliquent les incohérences.

\section{La seconde typologie oul'opposition entre société militaire et société industrielle}

Cette opposition peut être interprétée en posant la question à deux niveaux : le problème de la finalité, le problème de la définition sociologique de l'espace qui sépare les individus et la société.

\section{La question des fins}

Nous avons montré comment l'opposition entre société simple et société composée pouvait être comprise comme axiome théorique, et quels étaient ses liens avec le modèle organiciste. Mais cette typologie laisse de côté un ensemble de questions qu'il convient de résoudre pour qui prétend définir sociologiquement la société. L'opposition entre société simple et société composée 
n'avait pas pour but, par exemple, de répondre à la question de la finalité : la société doit-elle remplir une fin particulière qui se distingue de celle des actions individuelles ? Cette question devient nécessaire lorsque Spencer pose le problème de la formation de l'autorité politique. Chercher à comprendre comment elle s'établit c'est poser la question des fins, et la réponse sera encore donnée sous forme de typologie.

L'hypothèse sera la suivante. La société militaire, définie par le principe de la coopération obligatoire et par un système de droit «à régulation positive », correspond à un modèle de société finalisée du point de vue de la totalité. Cette totalité, son principe et son contenu sont incarnés et mis en œuvre par la classe militaire. La société industrielle, définie par le.principe de coopération indirecte libre et par un droit à « régulation négative », est une société conçue comme espace des interactions individuelles sans finalité superposée émanant de la totalité. Il n'y a de finalité que celle qui mobilise les actions individuelles.

Nous allons voir qu'il s'agit d'opposer le point de vue holiste, qui définit la société militaire, au point de vue individualiste, qui définit la société industrielle. Nous retrouvons ici l'hétérogénéité dont nous faisions l'hypothèse: le recours à un modèle théorique de type organiciste pour la société militaire (que nous pouvons ramener à un primat de la totalité sur les parties), à un modèle théorique de type individualiste pour définir la société industrielle; penser l'interaction à partir des parties composantes selon un schéma de type « mécaniste ${ }^{24}$, ou encore individualiste.

Caractérisons plus exactement ces deux types de société. La société militaire ne laisse aucun espace de liberté entre volonté commune et volonté individuelle. Ceci est classiquement évoqué dans le principe de fusion de l'individualité avec le tout : il n'y a pas d'autonomie. Le tout détermine les parties, et, dans la pratique, le caractère contraignant de la totalité s'impose par

24. En effet, Spencer va nous proposer une étude de la structuration de la société sur la base du modèle mécanique d'une interaction des forces, l'équilibre se définissant par une résultante principale. Nous pourrions ici faire une autre analogie avec Leibniz, celle du passage des volontés antécédentes aux volontés conséquentes, dans les Essais de Théodicée. 
l'obligation de la coopération, laquelle exprime une société politiquement hiérarchisée où l'intégration au tout est liée aux clivages et oppositions de classes. Donc, une société « finalisée », hiérarchisée et dont la logique de développement repose sur les conflits entre sociétés ${ }^{25}$. La finalité première, celle de la société entière, contient celles, secondaires et individuelles, des actions particulières. Dans un tel cadre, le droit prescrit ce qui doit être fait - positivité - : la particularité de l'action individuelle est tout entière contenue dans l'expression juridique de la loi qui, ellemême, n'est que la traduction institutionnelle de la volonté d'une minorité au pouvoir - les militaires qui s'identifient à la société.

Qu'en est-il de la société industrielle? Le jeu de la coopération indirecte libre présuppose, à titre de condition de possibilité, l'immanence du principe de constitution du tout. Cette émanantisation $^{26}$ est repérable à travers tous les changements significatifs qu'apporte avec elle la mise en place de cette société. C'est l'objet principal du chapitre XVIII ${ }^{\circ}$ de la cinquième partie des Principes. Soulignons le caractère essentiellement déductif de cette construction qui dessine, a contrario, les contours de la société industrielle comme idéal-type et non comme description du réel.

Toutefois, la disparition de la nécessité du travail coopératif obligatoire pose un problème central : comment penser la solidarité du tout ? Car avec la société industrielle, la cohésion de l'ensemble ne peut plus être pensée comme détermination du tout sur la partie. Spencer va avoir recours à un autre modèle théorique tout à fait conforme à la représentation de la société comme ensemble de monades : c'est le principe de l'harmonie préétablie. En effet, comment penser que la société peut tenir en un tout, dès lors que ce qui est posé comme premier est la partie, c'est-à-dire l'individu ? Nous pouvons dire que le principe de l'harmonie

25. On pourra lire avec attention le $\S 55$ d du t. III sur ce point, par exemple : « Bref, sous le régime militaire, lindividu est la propriété de l'Etat. Si la conservation de la société est la fin principale, la conservation de chaque membre est la fin secondaire quill faut assurer dans lintérêt de la principale. »

26. Qui s'oppose ici à transcendant : la société n'est pas formée ni ne tient ensemble grâce à un principe hors-la-société, mais par des facteurs qui lui sont internes. Ce n'est plus par Dieu que tient la société, par exemple, mais ce sont les hommes qui font leur société. 
préetablie va rendre possible la saisie théorique de l'ordre spontané. Remarquons, au passage, que nous ne sommes pas loin de la métaphore smithienne de la Main Invisible : « Si chacun pourvoit complètement à son propre bien-être et à celui de ses rejetons, le bien-être de la société est implicitement réalisé » [§ 564]. Ainsi, la représentation spencérienne de la société industrielle devient tout à fait conforme à celle induite par l'adoption du modèle théorique de type individualiste : la société est un ensemble de monades ; leur solidarité ne peut être pensée que sur le mode de l'ordre spontané grâce au principe de l'harmonie préétablie. Il y a donc bien renversement dans l'ordre de détermination totalité / parties, et, en même temps, c'est le point de vue qui bascule : du point de vue holiste au point de vue individualiste.

Dans ce cadre le problème devient celui de la définition du rôle et de l'étendue de l'intervention de l'organisation politique dans la société. Reprenant à son compte les arguments de A. 5 mith ${ }^{27}$, Spencer affirme « que le but que l'action publique a encore à atteindre, c'est de maintenir l'action dans certaines limites $\gg$ [\$ 564]; plus loin, abordant les conséquences de la disparition de la hiérarchie, il affirme que « la société a pour devoir essentiel de défendre l'individualité de ses membres let doncl, qu'une condition essentielle du type industriel veut que l'individualité de chaque homme ait le champ libre autant que le comporte la liberté de l'individualité des autres hommes » [§ 564]. Alors, la justice signifiera « la conservation des relations normales entre les actes et les résultats ». Comment comprendre ce raccourci de l'argument?

Dès lors que la société est posée comme espace des interactions individuelles où l'Etat n'a pas un rôle à jouer dans la définition d'une finalité pour la totalité, sa fonction s'oriente vers la légalisation des moyens. Le sens de l'intervention de l'État est celui de la prescription négative : « Ne fais pas cela! » le droit n’a

27. Diaconide, dans sa thèse sur la sociologie de Spencer, mentionne que celui-ci a eu connaissance des textes de Smith, et tout particulièrement de la Théorie des sentiments moraux, dans les années I840-1845. Il disait qu'il voulait s'en inspirer pour la rédaction de ses Principles of Ethics. E. Diaconide, Etude critique sur la sociologie de H. Spencer, Paris, Librairie générale de droit et de jurisprudence, 1938 . 
plus pour fonction éminente de légiférer sur les fins (positivité du droit dans la société militaire), mais d'arbitrer les moyens.

Parce que la loi devient médiation purement instrumentale, la régulation négative doit se limiter à prendre en charge la définition des conditions permettant à chacun d'agir sans entraver autrui. Sur ce plan, et sur celui-ci seulement, cette forme de régulation repose sur l'égalité : tous les individus sont égaux devant l'accès aux moyens pour satisfaire leurs intérêts.

Spencer déduit à tort la régression du droit comme médiation nécessaire au jeu des interactions individuelles. Sur ce point, Durkheim objectait que la mise en place des rapports d'échanges contractuels s'accompagnait d'un développement important du droit restitutif, lequel marquait une intervention de plus en plus grande de la société dans la réalisation des intérêts particuliers. Loin que la société industrielle provoque la disparition de toute forme de régulation juridique rappelant la prééminence du tout sur les parties, avec le droit restitutif, la société conserve un regard sur la finalité des actions individuelles, maintenant ainsi cette nécessaire cohérence du tout. De ce point de vue, ce droit peut s'interpréter comme développement d'un contrôle social dans le jeu des comportements.

Mais cette divergence des points de vue, au-delà des insuffisances de raisonnement de Spencer sur la place du droit dans les sociétés industrielles, illustre surtout le conflit des modèles théoriques mobilisés pour définir la société. Pour Durkheim, l'appréciation portée sur la finalité et l'importance du droit restitutif prend toute sa cohérence dans son postulat initial : le tout est plus que la somme des parties. Pour Spencer, le passage du droit positif au droit négatif est corrélatif d'un autre basculement : du primat de la totalité au primat de la partie. Ce renversement, tout à fait conforme à celui qu'il revendique dans son Introduction à la science sociale, achève d'établir sa conception libérale de la société industrielle.

De là il suit que la séparation du politique et de la sphère des intérêts individuels prendra la forme généralisée de l'espace marchand des échanges économiques.

Nous pouvons conclure sur ce point en affirmant qu'une des grandes différences entre Spencer et Durkheim est bien la 
définition du point de vue à partir duquel est saisi le social comme totalité. C'est cela que nous devons maintenant éclairer.

\section{Société militaire / société industrielle : comment est pensé l'espace qui sépare individus et société ?}

Ce qui a été dit permet d'affirmer que le principe par lequel est pensée la cohérence de la totalité dans la société industrielle renvoie au modèle individualiste de l'harmonie préétablie. Partant de l'individu envisagé comme monade une et résolument distincte des autres, il est nécessaire de postuler un principe de totalité sous une forme précise : de la différenciation naît l'interdépendance. Cette dernière est liée, chez Spencer, à la loi physiologique de la division du travail. Cela revient à dire que le mouvement qui se dessine de l'individu vers la société peut être défini, théoriquement, comme principe de dépendance fonctionnelle des parties au tout: c'est la relation sociale dont la première forme générale est l'échange ${ }^{28}$.

Mais tout cela entraîne une autre conséquence qui, selon nous, confirme l'hypothèse que nous faisions de l'usage méthodologique des modèles théoriques chez Spencer : il n'y a pas de confusion entre modèle et objet modélisé. C'est dire qu'il s'agit bien d'un point de vue, au sens où Weber le définit. C'est d'ailleurs à cette condition seulement qu'il peut y avoir une positivité de la connaissance sociologique. Ce point de vue s'élabore de la manière suivante: il y a une relation autonome qui relie individus et totalité (immanence de la loi d'évolution et des principes de développement de la société), et cette relation se démarque à la fois de la logique des actions individuelles et du point de vue que l'on adopte sur elle.

Nous pouvons maintenant reformuler le principe général sur lequel repose toute la seconde typologie.

I. La société industrielle se caractérise par le rejet de toute finalité sociale, elle implique une interdépendance « autonome » entre les parties, ce qu'il appellera marché :

28. Cette problématique n'est pas nouvelle, on la retrouve dans toute la littérature des Moralistes anglais de la tradition de l'école anglo-écossaise, à commencer par A. Smith. 
Il n'est plus question maintenant pour l'État de fixer des prix ni de prescrire des méthodes $[. .$.$] ; les citoyens font leurs affaires de la$ façon qui leur semble la meilleure l... ; comment leur activité industrielle s'est-elle ajustée aux nécessités des circonstances? C'est grâce à un appareil internoncial par le moyen duquel les divers organes industriels rencontrent les uns chez les autres des stimulants ou des obstacles par suite de la hausse ou de la baisse de la consommation I...l. Cela veut dire qu'à côté de l'appareil régulateur politique il s'est formé un appareil régulateur industriel qui remplit sa fonction coordinatrice d'une maniere independante, c'est-à-dire, en réalité, un plexus de ganglions, lop. cit., t. II, p. 128-ı2g].

Ce qui est décrit ici n'est autre que le principe de marché au sens large par lequel se réalise l'émanantisation et l'autonomisation du principe régulateur de la société industrielle. Ce marché est le principe ordonnateur du tout qui fait tenir ensemble les parties sans imposer de finalité particulière autre que celle qui guide les individus.

2. Il devient clair que la société militaire peut se comprendre, outre son orientation belliciste, comme société qui ne connaît pas encore d'autonomisation du principe régulateur de la sphère des activités individuelles. Elle est orientée, guidée par un principe de totalité finalisée qui lui échappe. De là, résulte nécessairement la subordination de toutes les dimensions du social au politicomilitaire, à commencer par l'économique. En ce sens, la société militaire n'est pas la société libérale, et, dans l'ordre du discours, le sociologue est bien obligé de confondre finalité et principe de totalité.

\section{Conclusion}

L'analyse de la double typologie spencérienne des sociétés a permis d'en arriver aux résultats suivants.

- Il y a chez cet auteur une pluralité de modèles théoriques de mobilisés conformément à la complexité de l'objet étudié.

- L'oscillation entre modèle organiciste et modèle individualiste ne peut pas s'interpréter seulement comme inconséquence de l'auteur. La prédominance du recours à l'individualisme dans les typologies est en même temps l'expression d'une limite : l'organicisme ne permet pas de cerner exactement les particularités de la société moderne, et il est nécessaire de recourir à une représentation des phénomènes d'interaction au moyen d'un mécanisme ou 
de rapports entre forces - l'individualisme comme méthode. Aujourd'hui, nous parlerions de procédures d'agrégation, d'effets de composition.

- L'usage qui est fait du modèle organiciste n'est pas purement ontologique. Si les ambiguités sont nombreuses, elles ne parviennent pas à masquer que la nature des dissemblances retenues par l'auteur conduit déjà vers un changement de perspective : de l'organicisme à l'individualisme. C'est donc toute une critique implicite de l'organicisme comme moyen de représentation du social qui se trouve contenue dans les Principes. Remarquons finalement qu'une étude précise de la nature de cet organicisme pourrait bien nous conduire au résultat suivant : plus qu'un organicisme, ne s'agirait-il pas d'une représentation mécaniciste de la société que nous propose Spencer?

$\mathrm{Si}$, enfin, l'orientation évolutionniste de Spencer est non contestable, une analyse plus nuancée permettrait d'y voir l'indication du sens probable d'une histoire. La seconde typologie propose, certes, une vision historique normative, mais elle est aussi non déterministe : si le sens le plus avantageux de l'évolution des sociétés est le passage de la société militaire à la société industrielle, ce sens n'est ni nécessaire ni irréversible.

Université de Lyon 